International Journal of Medical Anesthesiology 2021; 4(3): 110-114

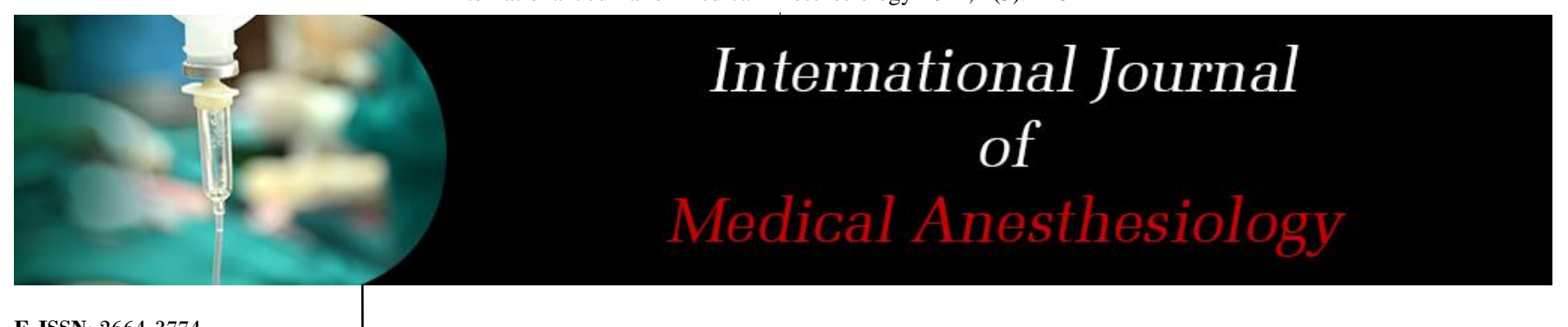

E-ISSN: 2664-3774

P-ISSN: 2664-3766

www.anesthesiologypaper.com

IJMA 2021; 4(3): 110-114

Received: 03-04-2021

Accepted: 19-05-2021

Dr. Sneha Srinivas

Resident Doctor, Department of Anaesthesiology, Srmsims,

Bareilly, Uttar Pradesh, India

Dr. Gaurav Misra

Assistant Professor,

Department of

Anaesthesiology, Srmsims,

Bareilly, Uttar Pradesh, India

Dr. Richa Chandra

Associate Professor,

Department of

Anaesthesiology, Srmsims,

Bareilly, Uttar Pradesh, India

Corresponding Author:

Dr. Gaurav Misra

Assistant Professor,

Department of

Anaesthesiology, Srmsims,

Bareilly, Uttar Pradesh, India

\section{Fascia Iliaca compartment block with $0.25 \%$ levo- bupivacaine versus intravenous fentanyl in patients with fracture of femur}

\author{
Dr. Sneha Srinivas, Dr. Gaurav Misra and Dr. Richa Chandra
}

DOI: $\underline{\text { https://doi.org/10.33545/26643766.2021.v4.i3b.289 }}$

\begin{abstract}
Introduction: Femoral neck fractures are often encountered in the elderly who may have many concurrent medical conditions. These patients are treated by surgery, more commonly under spinal anaesthesia. But the patients experience a significant amount of pain during positioning for administration of spinal anaesthesia. To alleviate this pain during positioning, multiple intravenous analgesics have been used. Recently regional blocks have gained popularity for the same purpose. Fascia iliaca compartment block (FICB) is one such safe alternative.

Method: 60 patients were divided into 2 groups of 30 each by computer- generated number sequence.

Group 1: Received the Fascia Iliaca compartment block with $20 \mathrm{ml}$ of $0.25 \%$ levo-bupivacaine 15 minutes before the subarachnoid block.

Group 2: Received Intravenous fentanyl at $1 \mathrm{mcg} / \mathrm{kg}$ single dose 15 minutes before the subarachnoid block.

Result: The mean VAS of group 1 was lower than that of group 2. The difference in VAS after the block and after the drug was statistically significant.

Conclusion: Fascia iliaca compartment block is better than fentanyl for providing analgesia during patient positioning in patients of fracture femur.
\end{abstract}

Keywords: Fascia iliaca compartment block, analgesia, fracture femur

\section{Introduction}

Femoral neck fractures are often encountered in the elderly who may have many concurrent medical conditions, commonly on polypharmacy. Fracture femur involving neck, shaft or distal femur causes severe pain and distress to the patient and needs surgical intervention. Subarachnoid block is generally given in these patients to provide anaesthesia. However, this needs the patient to be positioned ideally for the anaesthesia to be most effective. But positioning of the patient might be difficult due to intense pain caused by overriding of fracture ends ${ }^{[1]}$.

E.R. Viscusi et al. emphasized that pain left untreated may have significant physical and psychological effects on the patient, may delay operative management and complicate hospital stay ${ }^{[2]}$. Hence peri-operative pain management is crucial. While intravenous fentanyl has been used to provide analgesia in patients undergoing lower limb surgeries, peripheral nerve blocks have emerged as an effective mode of analgesia in these patients.

Peripheral nerve blocks like the femoral nerve block, 3 in 1 block and the Fascia Iliaca compartment block (FICB) are being used to provide analgesia and to avoid the side effects of opioids.

It has been demonstrated that the fascia iliaca compartment block, when given preoperatively provides analgesia in fractured femur during the sub arachnoid block as well as the post-operative period ${ }^{[3]}$.

Fascia iliaca blocks provide a safe, cheap and effective form of pain relief for patients with neck of femur fractures, and for those with femoral shaft fractures ${ }^{[4-6]}$.

Fascia iliaca compartment block (FICB) is an anterior approach to the lumbar plexus and was first described by Dalensin in $1989^{\text {[7] }}$. It is administered in the suprainguinal part of the femoral course.

This study compares the fascia iliaca block and intravenous fentanyl in patients undergoing surgery for fracture femur. 


\section{Aim and Objectives}

- To compare the efficacy of Fascia Iliaca Compartment Block with levo-bupivacaine versus intravenous fentanyl for analgesia to facilitate better positioning for spinal anaesthesia.

- To compare the haemodynamic parameters in the two groups.

- To compare complications if any - of either the block or intravenous fentanyl.

\section{Materials and Methods}

This study was conducted in a tertiary care centre after obtaining clearance from the institutional ethical committee.

\section{Inclusion Criteria}

1. Patients who give written informed consent.

2. Patients aged 18-80 years old.

3. Patients belonging to ASA grades 1 and 2 .

Patients undergoing surgery for fracture femur and willing to be part of the study.

\section{Exclusion Criteria}

1. Patients refusing to take part.

2. Patients with morbid obesity, hemorrhagic diathesis, peripheral neuropathy, psychiatric disorders, and infection at injection site.

3. Patients with allergy to local anaesthetics.

4. Patients on previous opioid therapy.

5. Patients with polytrauma.
Patients who fulfilled the inclusion criteria were explained about the procedure in detail and were divided in Group1/Group2 by a computer generated number sequence and were contained in sequentially numbered opaque envelopes to ensure blinding.

A prospective double blind study was conducted among 60 healthy ASA 1 and 2 patients in SRMS IMS, who were divided into 2 groups with 30 patients each.

Pre anaesthetic checkup was done, routine and special investigations were carried out depending on the history and clinical examination of the patient.

All the patients who gave consent were explained about the regional anaesthesia and VAS score preoperatively (and subjective response on the basis of patient satisfaction was noted).

They were assessed by investigations e.g., CBC, RBS, S. Urea, S. Creatinine, LFT, PT/ APTT, ECG, CXR, 2 D Echo if required.

Patients were kept Nil by mouth for 6 hours prior to surgery. In O.T, after patient' s arrival, standard monitor' s e.g., ECG, SpO2, NIBP, HR were attached.

All patients were preloaded with $500 \mathrm{ml}$ of RL, after securing an IV line. Inj. Ondansetron (0.15 $\mathrm{mg} / \mathrm{kg})$ intravenously was given as premedication to all the patients.

Group 1: Received the Fascia Iliaca compartment block with $20 \mathrm{ml}$ of $0.25 \%$ levo-bupivacaine 15 minutes before the subarachnoid block.

Group 2: Received Intravenous fentanyl at $1 \mathrm{mcg} / \mathrm{kg}$ single dose 15 minutes before the subarachnoid block.

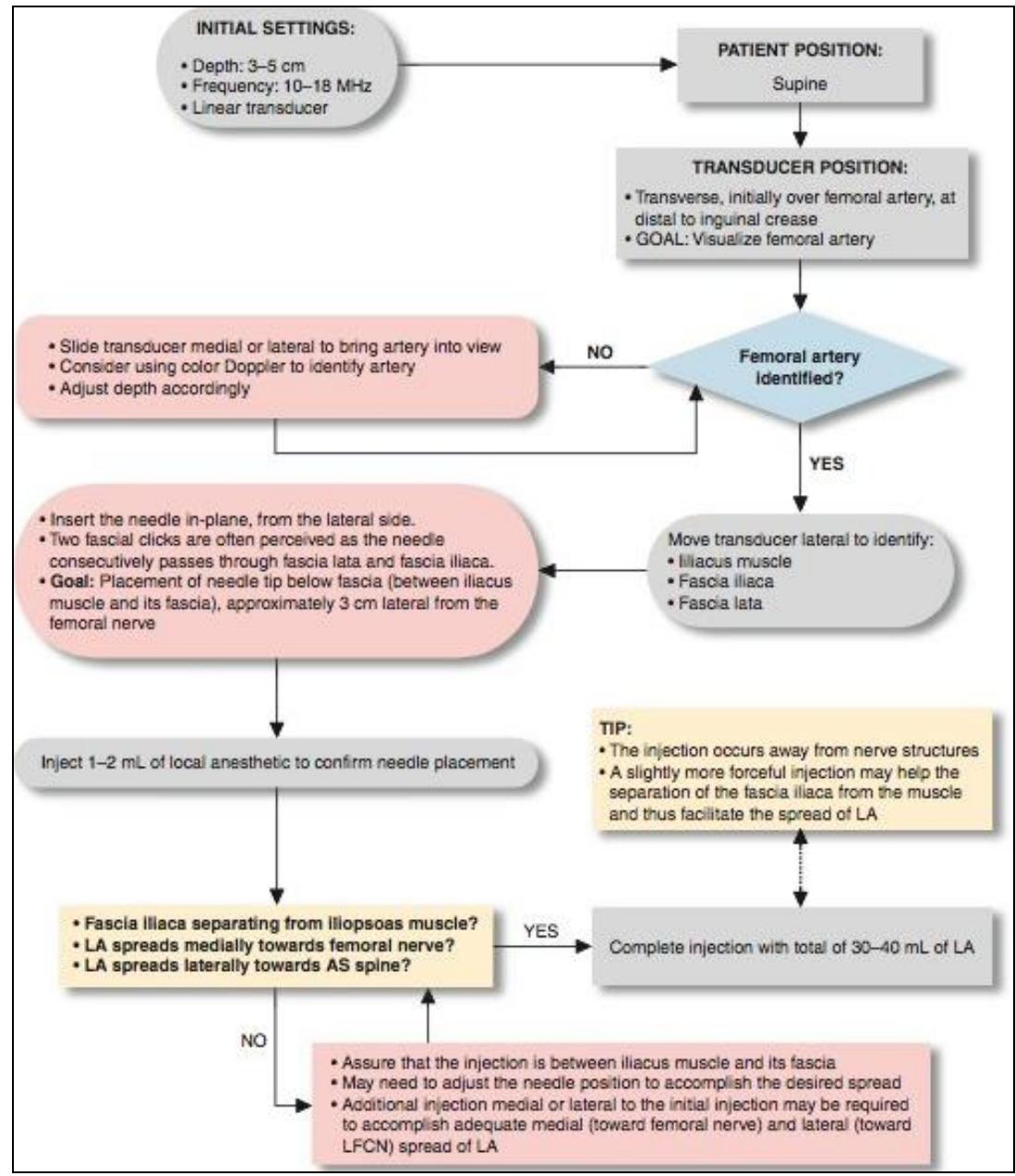

Fig 1: Ultrasound-guided fascia iliaca black 

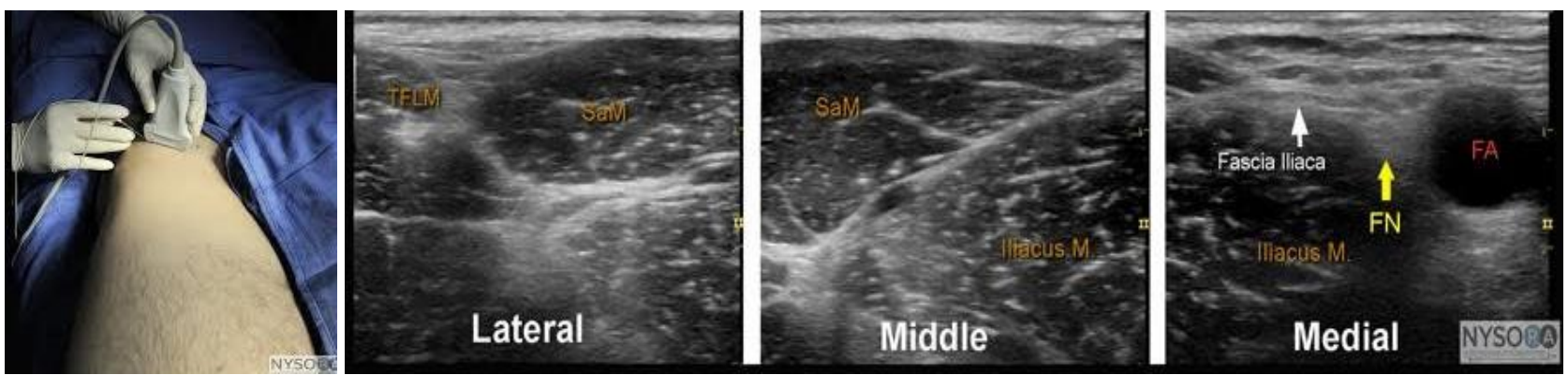

Fig 2: Probe position and fascial planes on ultrasound

A proper injection will result in the separation of the fascia iliaca by the local anesthetic in the medial-lateral direction from the point of injection.

The injection was given making sure there is no pain or paraesthesia on injection and that there is no resistance to injection. Heart rate, NIBP, $\mathrm{SpO} 2$, respiratory rate were recorded before the block/ before Inj. Fentanyl and at five minute intervals throughout the procedure.

The analgesia provided by either of the modes was subjectively assessed by using Visual analogue scale scores before the block/IV analgesia and after the block/IV analgesia at 5 minutes and thereafter every 2 minutes upto 15 minutes and the final difference in VAS scores was also noted.

The quality of patient positioning for administering spinal anaesthesia was recorded by another anaesthesiologist who was blinded to the mode of analgesia with scores of 0- 3 .

0 - not satisfactory

1. satisfactory

2. good

3. optimal

Time to perform spinal anaesthesia was recorded (time from beginning of positioning to end of spinal anaesthesia block).

Results: The observation and result of fascia iliaca compartment block and IV fentanyl was compared and statistical analysis was done. Data was managed in an excel spreadsheet. Quantitative values were assessed for approximately normal distribution. Each of those variables was summarized by mean and SD. For comparing the two main groups Paired t test was applied.

SPSS statistical software was used for data analysis. In this study $\mathrm{p}$ value $<0.05$ has been considered statically significant.

Table 1: Demographic factors

\begin{tabular}{|c|c|c|c|}
\hline & Group I & Group II & p Value \\
\hline Age in years & $44.6+17.12$ & $42.4+15.16$ & 0.05 \\
\hline Height in cm & $154.22+3.8$ & $155.7+3.08$ & 0.78 \\
\hline Weight in kg & $61.3+6.02$ & $62.8+7.08$ & 0.77 \\
\hline BMI & $24.7+6.05$ & $24.99+5.14$ & 0.33 \\
\hline
\end{tabular}

Demographic data in both groups are comparable because $p$ value is not significant.

Table 2: Surgeries performed

\begin{tabular}{|c|c|c|}
\hline Surgeries & Group I & Group II \\
\hline PFN & 12 & 14 \\
\hline DHS & 6 & 7 \\
\hline IMNIL & 8 & 4 \\
\hline CC SCREW FIXATION & 4 & 5 \\
\hline
\end{tabular}

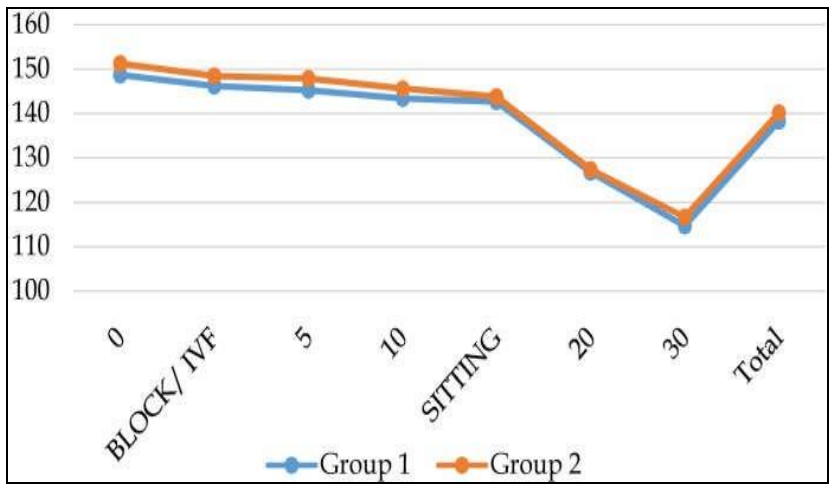

$\rightarrow$ Group $1 \rightarrow-$ Group 2

Fig 3: Comparison of systolic blood pressure in the two groups. 


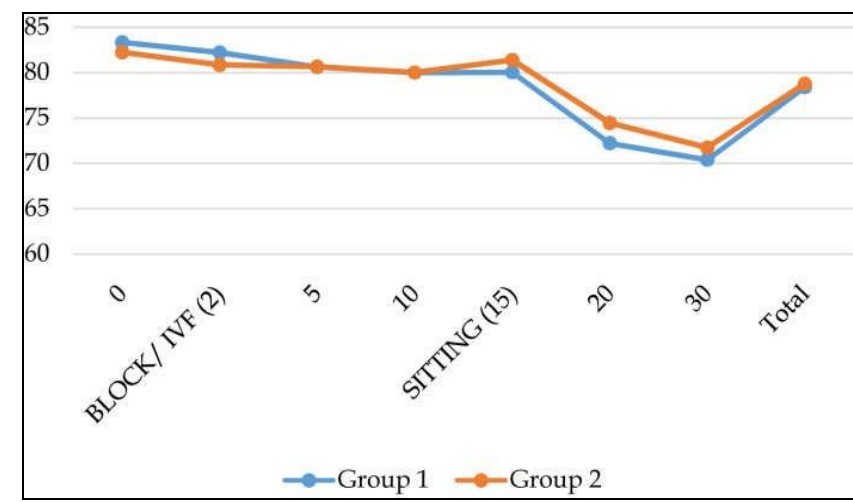

Fig 4: Comparison of diastolic blood pressure in the two groups

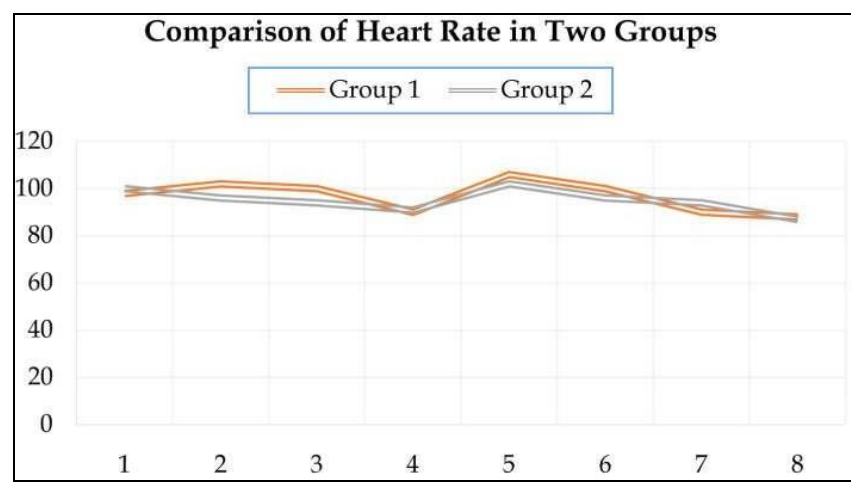

Fig 5: The difference in the haemodynamic parameters in the two groups was not found to be statistically significant.

Table 3: Duration to perform block

\begin{tabular}{|c|c|c|c|}
\hline Groups & Mean Time & Sd & P Value \\
\hline Group I & 6.3 & 0.3 & \\
\hline Group II & 8.67 & 0.5 & 0.0001 \\
\hline
\end{tabular}

Time taken to give SAB was compared in the two groups using student-t-test. And the difference in time was found to be statistically significant.

Table 4: VAS scores

\begin{tabular}{|c|c|c|c|}
\hline & Group I & Group II & P Value \\
\hline Difference In Vas & $5.05 \pm 1.64$ & $3 \pm 1.02$ & 0.0001 \\
\hline
\end{tabular}

The difference in the VAS scores was measured in both the groups and compared. The difference was statistically significant.

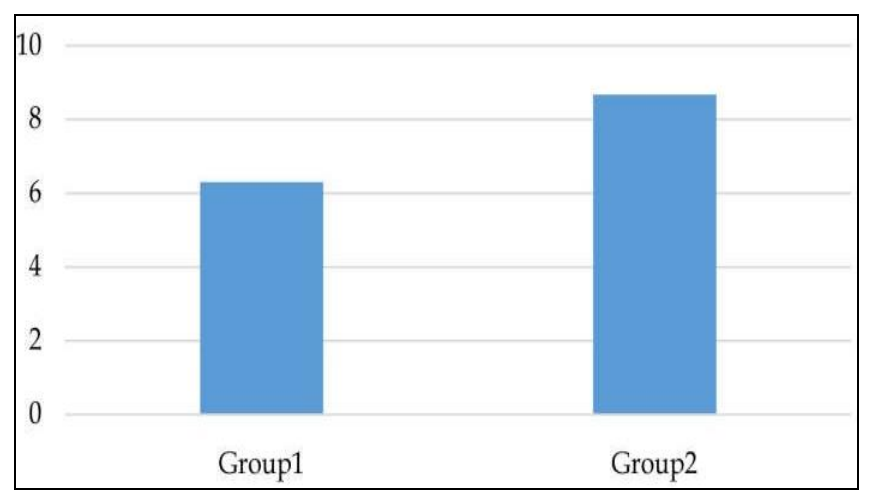

Fig 6: Time taken for $\mathrm{SAB}$ in two groups

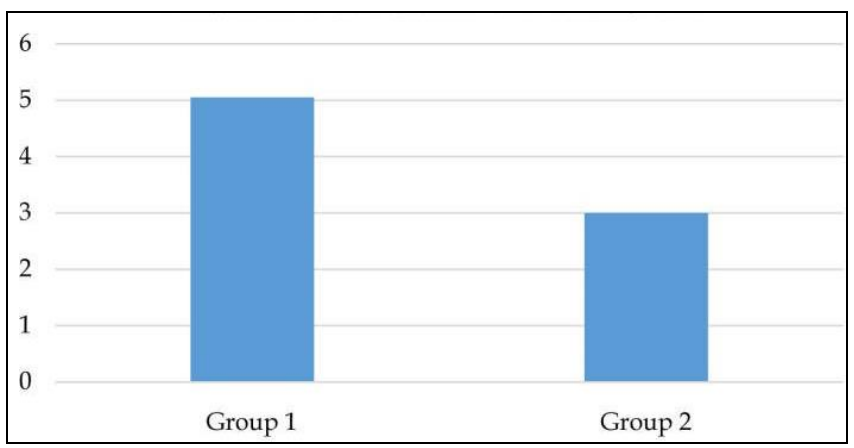

Fig 7: Comparison of difference of VAS in two groups

Table 5: Positioning scores

\begin{tabular}{|c|c|c|c|c|c|}
\hline $\begin{array}{c}\text { Positioning } \\
\text { Score }\end{array}$ & Group I & & Group II & & P Value \\
\hline 0 & 0 & 0 & 3 & 10 & \\
\hline 1 & 2 & 6.666667 & 5 & 16.66667 & \\
\hline 2 & 12 & 40 & 18 & 60 & \\
\hline 3 & 16 & 53.33333 & 4 & 13.33333 & 0.0024 \\
\hline
\end{tabular}

Ease of positioning was determined on the basis of score given to each patient using chi square test; for statistical analysis positioning score 0 and 1 was combined into one group and the data showed that the difference was statistically significant.

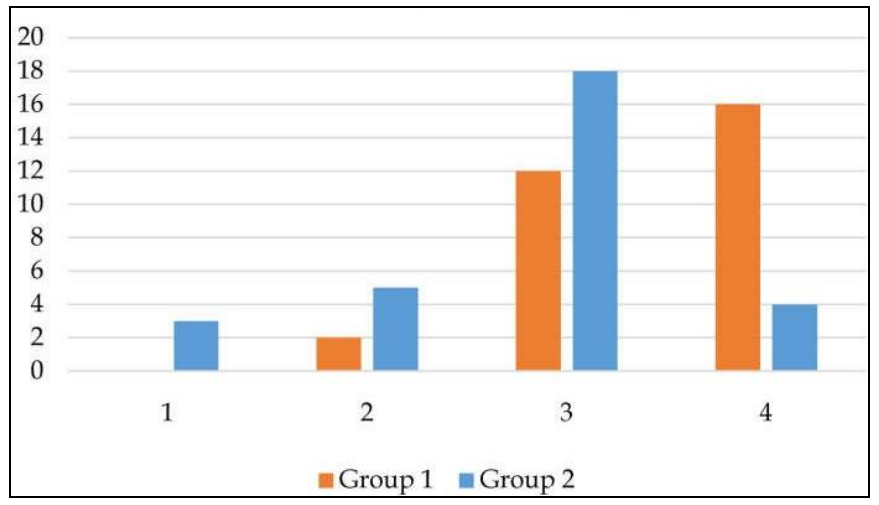

Fig 8: Comparison of sitting position in two groups

\section{Discussion}

Accidental application of fascia iliaca block by Dalens in 1989 while trying to block the lateral femoral cutaneous nerve helped anaesthesiologists learn and familiarize themselves with this technique.

It was found to be more effective than 3 in 1 block, cheaper and easier to perform.

Fracture femur is commonly associated with trauma more in the elderly due to coexisting osteoporosis and reduced bone mineral density but can occur in the young individuals as well.

Irrespective of the etiology leading to the fracture femur; our prime concern is to relieve patient from this painful situation and to help in making position of patient for regional anaesthesia.

Adequate analgesia is mandatory to be administered to the patient in emergency room before shifting the patient and in the perioperative period before positioning and fixing the fracture as pain arises from the periosteum of the bone 
which is very sensitive and it causes spasm of the thigh muscles and displacement of the fracture ends worsening the pain further.

Surgical fixation of the fracture is the definitive treatment and it also reduces pain.

Adequate perioperative analgesia reduces morbidity and mortality further and thus helps in improving the quality of health care being provided.

Regional anaesthesia is preferred over general anaesthesia for the fixation of fracture femur as it is associated with lesser incidence of postoperative pulmonary complications, lesser ICU stay and lesser morbidity.

This study tried to compare FICB with intravenous injection fentanyl so as to determine which is better in providing adequate perioperative analgesia and proper positioning of the patient.

Demographically both the study groups were found to be comparable. The type of fracture and surgery performed in the two groups was also similar. The distribution of patient on the basis of ASA physical status was also comparable.

Analgesic efficacy of the two techniques was determined on the basis of VAS score.

DG Monzon et al. compared parenteral NSAIDS with fascia iliaca block in elderly patients and found FICB to have rapid onset and was effective up to 8 hours to control post hip fracture pain.

Yun M.J. et al. in their study compared analgesia between FICB with a catheter using Ropivacaine and IV Alfentanil before positioning the patient in lateral position for SAB.

$60 \%$ patients got incomplete block, even 20 minutes after the block, there VAS scores ranged between 1 and 4 median 2 during positioning and they did not request supplementary analgesics. The mean VAS scores in FICB group was 2 versus 3.5 in the Alfentanil group and the difference in the two groups was statistically significant.

In another study conducted by Sia $\mathrm{S}$ and colleagues; analgesic efficacy of femoral nerve block with lidocaine versus IV fentanyl before positioning for spinal anaesthesia in fracture shaft of femur only. They found a statistically significant reduction in VAS scores after femoral nerve block compared to IV fentanyl group.

In our study the mean VAS score in both the groups of study was 7.9 and there was a drop in VAS Scores of both the groups after using FICB and IV fentanyl. This drop in VAS score was statistically significant in both the groups and more so in the FICB group.

Positioning was determined on the basis of use of a score of 0-3 depending on the ease or comfort with which the patient sat for SAB. The score was given by the anesthesiologist who was blinded to the technique being used.

The rise in blood pressure during patient positioning was higher in IV fentanyl group as compared to FICB which could be due to better analgesia provided by FICB.

The patients in both the groups remained hemodynamically stable throughout the procedure.

FICB was not associated with any complication in our study. Patients receiving IV fentanyl had side effects like drowsiness and nausea but it was not clinically significant. Patients in both the groups were haemodynamically stable.

\section{Conclusion}

In this study we compared FICB with IV fentanyl for perioperative analgesic effect and patient positioning with fracture femur for $\mathrm{SAB}$ and FICB was considered to be more efficient than IV fentanyl.

This was concluded on the basis of reduced VAS score, better sitting position, lesser time required to perform the sub arachnoid block.

Thus we conclude FICB is better as an option over IV fentanyl in reducing pain in patients with fracture femur.

\section{References}

1. Yun MJ, Kim YH, Han MK, Kim JH, Hwang JW, Do SH. Analgesia before a spinal block for femoral neck fracture: fascia iliaca compartment block. Acta Anaesthesiol Scand 2009;53:1282-287.

2. Viscusi ER, Pappagallo M. A review of opioids for inhospital pain management, Hospital practice 2012;40(1):149-159.

3. Nicolai B Foss, Billy B Kristensen, Morton Bundgaard, Mikkel Bak et al. Fascia iliaca compartment blockade for acute pain control in hip fracture patients. Anesthesiology 2007;106:773-8.

4. Vassiliadis J, Hitos K, Hill CT. Factors influencing prehospital and emergency department analgesia administration to patients with femoral neck fractures, Emergency Medicine 2002;14(3):261-266.

5. Hanna L, Gulati A, Graham A. The Role of Fascia Iliaca Blocks in Hip Fractures: A prospective CaseControl Study and Feasibility Assessment of a JuniorDoctor-Delivered Service, ISRN Orthopedics, 2014.

6. Mouzopoulos G, Vasiliadis G, Lasanianos N, Nikolaras G, Morakis E, Kaminaris M. Fascia iliaca block prophylaxis for hip fracture patients at risk for delirium: a randomized placebo-controlled study, Journal of Orthopaedics and Traumatology 2009;10(3):127-133.

7. Dalens B, Vanneuville G, Tanguy A. Comparison of the fascia iliaca compartment with 3-in-1 block in children. Anesth Analg 1989;69:705-13. 